\title{
Clinical utility of a novel natural killer cell activity assay for diagnosing non-small cell lung cancer: a prospective pilot study
}

This article was published in the following Dove Medical Press journal: OncoTargets and Therapy

\author{
Sue In Choi ${ }^{1, *}$ \\ Seung Hyeun Lee ${ }^{2, *}$ \\ ji-Young Park ${ }^{3}$ \\ Kyoung-Ah $\mathrm{Kim}^{3}$ \\ Eun Joo Lee' \\ Sang Yeub Lee' \\ Kwang Ho In' \\ 'Division of Respiratory and Critical \\ Care Medicine, Department of \\ Internal Medicine, Korea University \\ College of Medicine, Seoul, South \\ Korea; ${ }^{2}$ Division of Pulmonary, \\ Allergy and Critical Care Medicine, \\ Department of Internal Medicine, \\ Kyung Hee University School of \\ Medicine, Seoul, South Korea; \\ ${ }^{3}$ Department of Clinical Pharmacology \\ and Toxicology, Korea University \\ College of Medicine, Seoul, \\ South Korea \\ *These authors contributed equally \\ to this work
}

Purpose: Although decreased natural killer cell activity (NKA) has been observed in many solid cancers, clinical implication of NKA has been scarcely investigated in lung cancer. The objective of this study was to evaluate the potential of using NKA to support diagnosis of nonsmall cell lung cancer (NSCLC).

Materials and methods: We prospectively evaluated and compared peripheral blood NKA using a novel interferon-gamma releasing assay in healthy population $(n=40)$, patients with benign lung disease $(n=40)$, and those with NSCLC $(n=71)$. We explored the correlation between NKA and clinical parameters and assessed diagnostic performance of NKA for NSCLC using receiver operating characteristic curve analysis.

Results: Median NKA values in healthy population, patients with benign lung disease, and those with NSCLC were 1,364.2, 1,438.2, and $406.3 \mathrm{pg} / \mathrm{mL}$, respectively. NKA in NSCLC patients was significantly lower than that in the other two control groups (both $P<0.001$ ). At a cutoff value of NKA at $391.0 \mathrm{pg} / \mathrm{mL}$, the area under the curve was 0.762 (95\% CI: $0.685-0.838$, $P<0.001$ ), with a sensitivity of $52.3 \%$, a specificity of $91.0 \%$, a positive predictive value of $85.3 \%$, and a negative predictive value of $65.4 \%$ for the diagnosis of NSCLC. Multivariate analysis demonstrated that diagnosis of NSCLC is the only clinical parameter that was significantly associated with NKA $(P<0.001)$.

Conclusion: This pilot study showed that patients with low NKA were more likely to have lung cancer. Further studies are warranted in order to establish the clinical utility of NKA test for diagnosing lung cancer.

Keywords: lung cancer, natural killer cell, interferon-gamma, diagnosis, ELISA

\section{Introduction}

Natural killer (NK) cells are subpopulation of lymphocytes that play a major role in antitumor immunity. NK cells not only directly eliminate tumor cells by releasing cytolytic granules, but also stimulate adaptive immune response by secreting various cytokines against tumor cells. ${ }^{1} \mathrm{NK}$ cells are phenotypically defined by the expression of CD56 and lack of CD3 in their cell membrane. Human NK cells are divided into two subsets according to membrane density of CD16 and CD56. CD16 CD56 ${ }^{\mathrm{dim}}$ cells comprise the majority of NK cells and exhibit cytotoxic activity while $\mathrm{CD} 16^{-} \mathrm{CD} 56^{\text {bright }}$ cells account for the minority and secrete various cytokines, including interferongamma $(\mathrm{IFN}-\gamma){ }^{2}$ The latter can also acquire potent cytotoxicity after they are activated by proinflammatory cytokines. ${ }^{3}$ Studies have shown that NK cell activity (NKA) is important for antitumor immune surveillance by suppressing tumor metastasis and outgrowth. ${ }^{4,5}$ Interestingly, NKA is decreased in several human cancers, showing
Correspondence: Kwang Ho In Division of Respiratory and Critical Care Medicine, Department of Internal Medicine, Korea University College of Medicine, 73, Inchon-ro, Seongbuk-gu, Seoul 0284I, South Korea

Tel +8229205316

Fax +82 29292045

Email khin@kumc.or.kr hereby accept the Terms. Non-commercial uses of the work are permitted without any furcther permission from Dove Medical Press Limited, provided the work is properly attributed. For permission for commercial use of this work, please see paragraphs 4.2 and 5 of our Terms (https://www.dovepress.com/terms.php). 
negative correlation with tumor stage. ${ }^{6,7}$ Low NKA is associated with invasiveness of malignancy and high level of tumor recurrence and metastasis while high NKA is associated with favorable clinical outcome in various human cancers. ${ }^{89}$ A large epidemiologic study enrolling $>3,600$ Japanese general population with follow-up of 11 years has shown that low NKA is significantly associated with increased risk of cancer (all sites), suggesting the importance of immune surveillance by NK cells against cancer as well as the association between NKA and development of cancers. ${ }^{10}$

Traditionally, chromium $51\left({ }^{51} \mathrm{Cr}\right)$ release assay or CD107a degranulation assay has been used to measure NKA. However, these methods are complex, expensive, and time consuming because they require isolation of peripheral blood mononuclear cells (PBMC) or NK cells from whole blood. ${ }^{11}$ To overcome these difficulties, an innovative in vitro diagnostic device, NK Vue ${ }^{\circledR}$ (ATGen, Seongnam, Korea) assay, has been recently developed and commercially used for the measurement of NKA. It simply measures IFN- $\gamma$ secreted from NK cells as an indicator of NKA using a small volume of whole blood. ${ }^{12}$ To stimulate NK cells ex vivo, this assay uses Promoca ${ }^{\mathrm{TM}}$, an engineered recombinant protein that can activate NK cells in the whole blood during incubation for 20-24 hours. In contrast to ${ }^{51} \mathrm{Cr}$ release assay, which measures cytotoxic potential of $\mathrm{CD} 16^{+} \mathrm{CD} 56^{\mathrm{dim}}$ subset, this assay measures IFN- $\gamma$ released from whole NK cell population, including both $\mathrm{CD} 16^{-} \mathrm{CD} 56^{\text {bright }}$ and $\mathrm{CD} 16^{+} \mathrm{CD} 56^{\mathrm{dim}}$ subsets as well as the ability of these cells to amplify immune response to tumors. ${ }^{12}$ A few previous studies have demonstrated clinical utilities of this assay for the diagnosis or risk stratification of several cancers, including gastric, colorectal, and prostatic cancers. ${ }^{13-16}$ However, clinical implication of this assay in lung cancer patients has not been reported yet.

Previous studies have demonstrated impaired IFN- $\gamma$ production and NK cell cytotoxicity as well as the association between tumor-infiltrating NK cells subset CD57 and prognosis in lung cancer patients. ${ }^{17,18}$ Considering these findings, NKA might be useful for diagnosing or predicting prognosis of patients with lung cancer as for other tumors. Thus, the objective of this explorative study was to investigate associations of NKA measured by NK Vue ${ }^{\circledR}$ assay with clinical parameters in lung cancer patients and determine its clinical utility for lung cancer diagnosis.

\section{Materials and methods}

\section{Study subjects and specimens}

To compare NKA levels between lung cancer patients and cancer-free population, we prospectively enrolled healthy population, patients with benign lung disease, and non-small cell lung cancer (NSCLC) patients at Korea University Anam Hospital, between January 2016 and September 2017 and measured NKA for all groups. Healthy population included those who underwent general medical examination in our institution. They had no current evidence or previous history of cancer or active infection. Patients with benign lung disease were chosen among those who had asthma, bronchiectasis, COPD, idiopathic pulmonary fibrosis (IPF), pulmonary tuberculosis (TB), or pneumonia. Patients with NSCLC were those who had histologically confirmed NSCLC and underwent staging workups, including chest computed tomography, brain magnetic resonance imaging, and ${ }^{18} \mathrm{~F}$-fluorodeoxyglucose positron emission tomography. TNM staging was determined according to the seventh edition of International Association for the Study of Lung Cancer TNM staging system. Patients who took immunosuppressive drugs, including corticosteroids within the last 3 months and those who had autoimmune disorders were excluded. We reviewed electronic medical records to obtain demographic information, past medical or social history, and clinical data of all participants. This study protocol was approved by Clinical Research Ethics Committee of the Korea University Anam Hospital (approval number: MD15009). Written informed consent was obtained from all participants. This study was conducted in accordance with the Declaration of Helsinki.

\section{Measurement of NKA}

NKA was measured using NK Vue ${ }^{\circledR}$ assay as described previously. ${ }^{12}$ Briefly, $1 \mathrm{~mL}$ of fresh blood was collected using $\mathrm{NK} \mathrm{Vue}^{\circledR}$ tube (ATGen) and incubated at $37^{\circ} \mathrm{C}$ in a $\mathrm{CO}_{2}$ chamber for 20-24 hours. For patients with benign lung disease and those with NSCLC, blood samples were obtained before any treatment. The $\mathrm{NK} \mathrm{Vue}{ }^{\circledR}$ tube contains Promoca ${ }^{\mathrm{TM}}$, a patented recombinant protein that can activate NK cells in whole blood. Following incubation, cell-free supernatants were harvested, transferred into $1.5 \mathrm{~mL}$ conical tubes, and centrifuged at 13,000 rpm for 1 minute at room temperature. Supernatants were again transferred into 1.5-mL conical tubes and frozen at $-20^{\circ} \mathrm{C}$ for a period of up to 2 weeks. IFN- $\gamma$ levels in these supernatants were measured by ELISA. Prior to assay, tubes were thawed at room temperature and centrifuged at 13,000 rpm for 1 minute at room temperature. The supernatant was then immediately loaded onto ELISA plate. Absorbance value was measured at wavelength of $450 \mathrm{~nm}$. Concentrations of IFN- $\gamma$ were determined with a calibration curve and expressed as $\mathrm{pg} / \mathrm{mL}$. The measuring range was $0.1-4,000 \mathrm{pg} / \mathrm{mL}$. 


\section{Flow cytometry for NK cell subset distribution}

Reduction in $\mathrm{CD} 16^{-} \mathrm{CD} 56^{\text {bright }} \mathrm{NK}$ cells has been suggested as one of the mechanisms of decreased NKA. ${ }^{19}$ Thus, we assessed $\mathrm{CD} 16^{+} \mathrm{CD} 56^{\mathrm{dim}} / \mathrm{CD} 16^{-} \mathrm{CD} 56^{\text {bright }} \mathrm{NK}$ cell subset ratio for some subjects of this study to investigate whether altered NKA is attributable to change of this ratio. The first 20 subjects from each group were selected and their NK cell subset distributions were analyzed using flow cytometry. A 5-mL sample of whole blood was collected in a heparin tube and diluted with PBS in a 1:1 ratio. PBMCs were isolated by centrifugation from the diluted whole blood and overlaid on Histopaque ${ }^{\circledR} 1077$ (Sigma-Aldrich, Darmstadt, Germany) in a conical tube. These collected PBMCs were washed and resuspended in staining buffer. PBMCs were stained with Alexa Fluor488-anti-CD56, APC-anti-CD16, and PE-antiCD3 fluorochrome-conjugated monoclonal antibodies (BD Biosciences, Seoul, Korea). After the reaction was completed, the mixture was transferred to a conical tube and analyzed by BD FACSCalibur ${ }^{\mathrm{TM}}$ (BD Biosciences). CD16 ${ }^{+} \mathrm{CD} 56^{\mathrm{dim}}$ and $\mathrm{CD} 16^{-} \mathrm{CD} 56^{\text {bright }} \mathrm{NK}$ cells were gated from $\mathrm{CD} 3^{-} \mathrm{CD} 56^{+}$cell population and the ratio of $\mathrm{CD} 16^{+} \mathrm{CD} 56^{\mathrm{dim}} / \mathrm{CD} 16^{-} \mathrm{CD} 56^{\text {bright }}$ was determined.

\section{Statistical analyses}

Continuous variables are described as median and IQR while categorical variables are presented as frequencies and percentages. Comparisons of clinical parameters of each group were performed using nonparametric Mann-Whitney test or Kruskal-Wallis test with Bonferroni correction. Receiver operating characteristic (ROC) curves were analyzed to determine optimal cutoff values and diagnostic sensitivity as well as specificity. Spearman's correlation analyses were used to assess the relationship between NKA and distribution of NK cell subsets. Associations between clinical parameters and NKA were first evaluated by univariate analysis using log-rank test. Multivariate logistic regression analysis was then conducted with adjustment for parameters with $P$-values of $<0.3$ in the univariate analysis. $P$-values of $<0.05$ were considered significant. Statistical analyses were performed using SPSS 20.0 software for Windows (IBM, Chicago, IL, USA).

\section{Results}

\section{Characteristics of subjects}

Demographics and clinical characteristics of subjects in each group are summarized in Table 1. A total of 151 subjects were enrolled, including 40 healthy population, 40 patients with benign lung disease, and 71 with NSCLC. All were Koreans.
Their median age was 68.7 years (range, 51-80 years). Although there were no significant differences in the distribution of age or gender, the proportion of ever smoker was significantly lower in the healthy population compared with that in the other two groups $(P<0.05)$. Among subjects with benign lung disease, 14 (35\%), 13 (32.5\%), $4(10.0 \%), 4(10.0 \%), 3(7.5 \%)$, and $2(5 \%)$ patients had COPD, asthma, TB, IPF, bronchiectasis, and pneumonia, respectively. Among patients with NSCLC, 31 (43.7\%) were early staged (stage I or II) while $40(56.3 \%)$ were advanced staged (stage III or IV). Forty-two (59.1\%) patients had adenocarcinomas and 29 (40.9\%) had squamous carcinomas.

\section{Comparison of NKA in three groups}

Median values of NKA in healthy population, patients with benign lung disease, and those with NSCLC were 1,438.2 pg/mL (IQR: 672.0-2,378.8 pg/mL), 1,364.2 pg/mL (IQR: $486.2-2,405.4 \mathrm{pg} / \mathrm{mL}$ ), and $406.3 \mathrm{pg} / \mathrm{mL}$ (IQR: 61.6-984.3 pg/mL), respectively. The NKA in the NSCLC group was significantly lower than that in the other two groups (both $P<0.001$ ). There was no significant difference in NKA between the healthy population and patients with benign lung diseases (Figure 1).

\section{Association between NKA and clinical parameters}

Relationships between NKA and clinical characteristics in each group are summarized in Table 1. NKA was not significantly different according to gender, age, or smoking status in all study groups. In those with benign lung disease, there was no significant difference in NKA according to the diagnosis. In those with NSCLC, NKA showed decreasing trend toward advanced tumor stage: $1,098.9 \mathrm{pg} / \mathrm{mL}$ (IQR: $508.1-$ 1,715.5 pg/mL), 844.5 pg/mL (IQR: 280.8-2,093.8 pg/mL), $121.6 \mathrm{pg} / \mathrm{mL}$ (IQR: $40.0-442.8 \mathrm{pg} / \mathrm{mL}$ ), and $228.8 \mathrm{pg} / \mathrm{mL}$ (IQR: 40.0-507.9 pg/mL) in stage I, stage II, stage III, and stage IV, respectively. NKA was not significantly different between stage I and II, or stage III and IV. However, NKAs in those with stage III or IV lung cancer were significantly lower than in those with stage I or II disease (all $P<0.05$, Figure $2 \mathrm{~A}$ ). Similarly, NKA of advanced (stage III and IV) disease was significantly lower than that of early-stage (stage I and II) disease (158.2 vs $912.3 \mathrm{pg} / \mathrm{mL}, P<0.001$ ) (Figure 2B).

To determine which clinical parameters might affect NKA, we performed univariate analysis for whole study population using variables, including sex, age, smoking status, and the diagnosis of benign lung disease or NSCLC. Results are summarized in Table 2. Univariate analysis showed that the diagnosis of NSCLC was significantly associated with 
Table I Clinical characteristics of the study populations and NKA in three groups

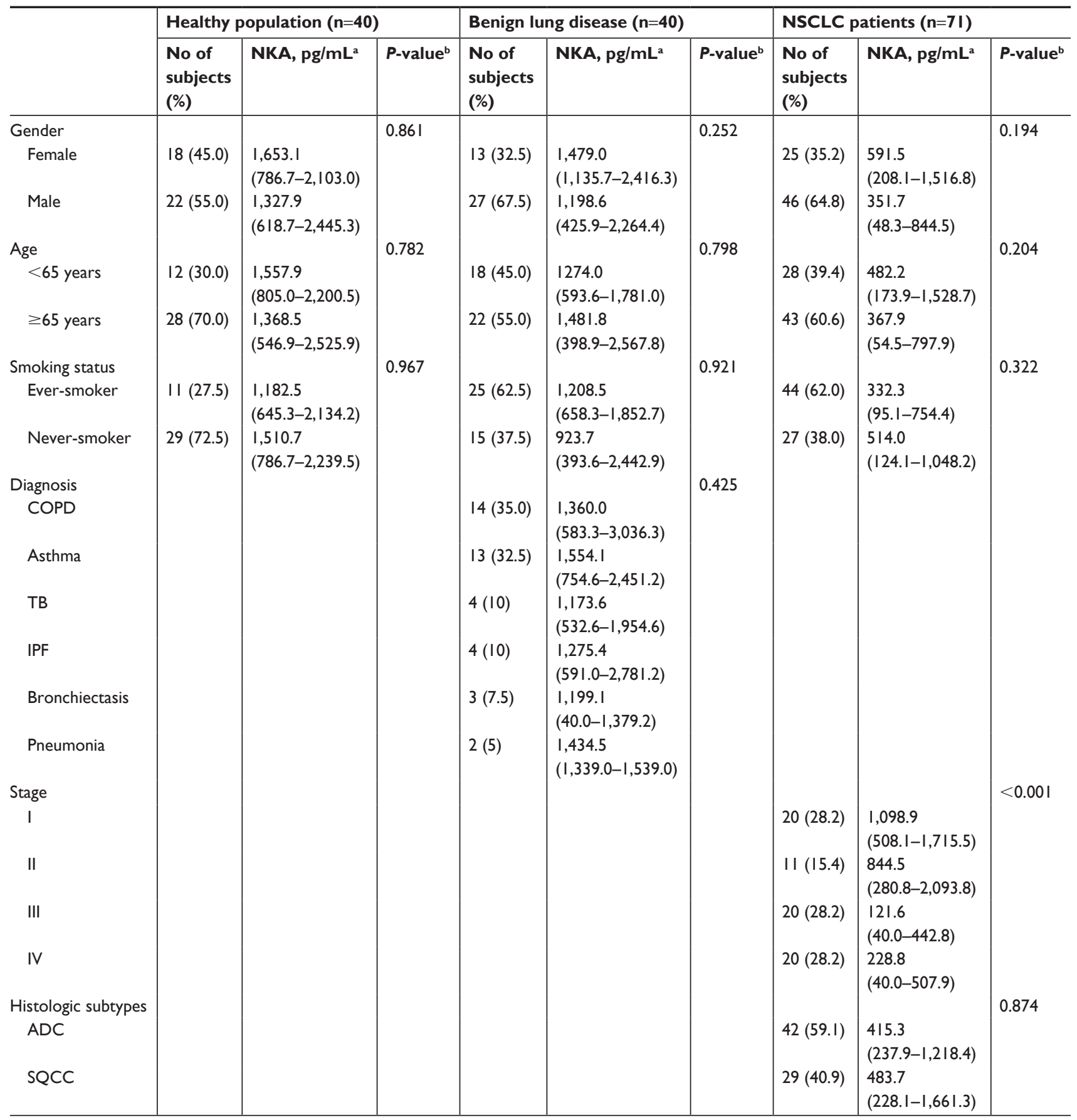

Notes: ${ }^{a} N K A$ was presented as median value with IQR. ${ }^{b} P$-values were calculated by nonparametric Mann-Whitney $U$ test or Kruskal-Wallis test.

Abbreviations: ADC, adenocarcinoma; IPF, idiopathic pulmonary fibrosis; NKA, natural killer cell activity; NSCLC, non-small cell lung cancer; SQCC, squamous cell carcinoma; TB, tuberculosis.

NKA $(P<0.001)$. In the multivariate analysis, the diagnosis of NSCLC was the only independent clinical parameter affecting NKA $(P<0.001)$.

\section{Comparison of NK cell subset distribution in three groups}

Representative flow cytometric data showed the distribution of total NK cell population represented as $\mathrm{CD}^{-} \mathrm{CD}^{-} 6^{+}$ cells (Figure S1A) and two major subsets, CD16 $56^{\mathrm{dim}}$ and CD16-CD56 $6^{\text {bright }}$, expressed as a percentage of total NK cells (Figure S1B). In overall population, CD16-CD56 $6^{\text {bright }}$ cells accounted for $2.8 \% \pm 2.5 \%$ of NK cells consistent with the results of a previous study on a Korean population. ${ }^{20}$ Median values of $\mathrm{CD} 16^{+} \mathrm{CD} 56^{\mathrm{dim}} / \mathrm{CD} 16^{-} \mathrm{CD} 56^{\text {bright }} \mathrm{NK}$ cell subset ratio in healthy population, patients with benign lung disease, and those with NSCLC were 54.2 (IQR: 27.9-53.4), 32.9 


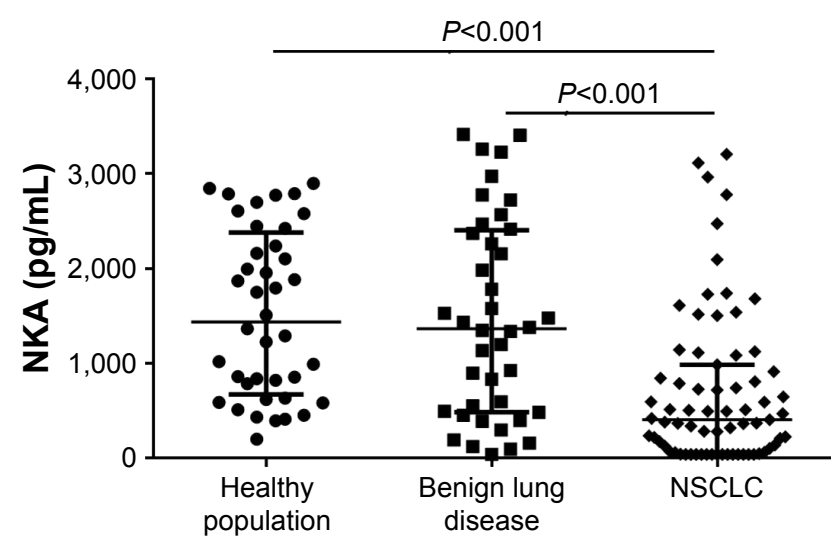

Figure I Comparison of NKA in three groups.

Notes: NKA is significantly decreased in NSCLC patients compared with that in healthy population and patients with benign lung disease (both $P<0.00 \mathrm{I}$ ). Bars denote median and IQR.

Abbreviations: NKA, natural killer cell activity; NSCLC, non-small cell lung cancer.

(IQR: 21.0-68.9), and 62.7 (IQR: 38.0-92.4), respectively. These ratios did not differ significantly among the three groups $(P=0.756)$. We also evaluated whether NKA might be correlated with $\mathrm{CD} 16^{+} 56^{\mathrm{dim}} / \mathrm{CD} 16^{-} \mathrm{CD} 56^{\text {bright }} \mathrm{NK}$ cell subset ratio in our study population. However, NKA was not correlated with such ratio (rho $=0.003, P=0.645)$ (Figure $\mathrm{S} 2$ ).

\section{Performance of NKA for the diagnosis of NSCLC}

The performance of NKA for the diagnosis of NSCLC was assessed using ROC curve analysis. With healthy population and patients with benign lung disease as reference, the area under the curve (AUC) was 0.762 (95\% CI: 0.685-0.838, $P<0.001)$. At a cutoff value of $391.0 \mathrm{pg} / \mathrm{mL}$, NKA had a sensitivity of $52.3 \%$, a specificity of $91.0 \%$, a positive predictive value of $85.3 \%$, and a negative predictive value of $65.4 \%$ for the diagnosis of NSCLC (Figure 3A). With benign lung disease group as reference, AUC was 0.735 (95\% CI: $0.638-0.830, P<0.001)$ with a sensitivity of $50.8 \%$ and a specificity of $89.1 \%$ (Figure 3B). With healthy population as reference, the AUC was slightly increased to 0.798 (95\% CI: $0.706-0.872, P<0.001)$ with a sensitivity of $53.6 \%$ and a specificity of $92.8 \%$ (Figure $3 \mathrm{C}$ ).

\section{Discussion}

This present pilot study showed that NKA was significantly decreased in NSCLC patients compared with that in healthy population or patients with benign lung diseases. At a cutoff level of $391.0 \mathrm{pg} / \mathrm{mL}$, NKA could discriminate NSCLC patients from other populations without lung cancer with high specificity. In addition, diagnosis of NSCLC was the only independent factor associated with NKA after adjusting for various clinical parameters. To the best of our knowledge, this is the first study to demonstrate decreased NKA in lung cancer patients measured by a novel IFN- $\gamma$ releasing assay and identify the potential usefulness of this assay as a supportive method for diagnosis of NSCLC.

Previous studies have reported the decreased NKA in a variety of human malignancies and the association between altered NKA and prognosis in various types of cancers., ${ }^{4,14,15}$ Thus, NKA might be useful as a potential prognostic or diagnostic biomarker in various tumors. To measure NKA, the current standard method is ${ }^{51} \mathrm{Cr}$ release assay or flow cytometry-based assays to reveal the cells' cytotoxic activity or ability to produce cytokines. However, those methods are labor-intensive and require long turn-around time. ${ }^{11}$ Thus, they are unsuitable for daily clinical practice. Measurement of IFN- $\gamma$ secreted from NK cells has been considered as a

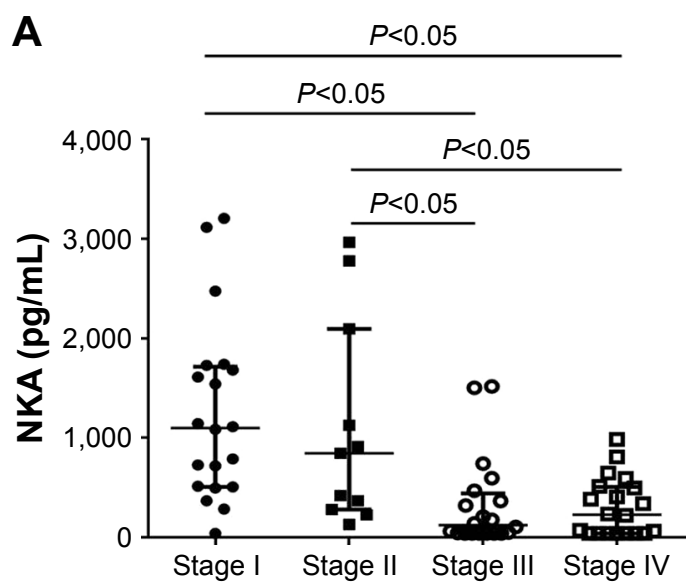

B

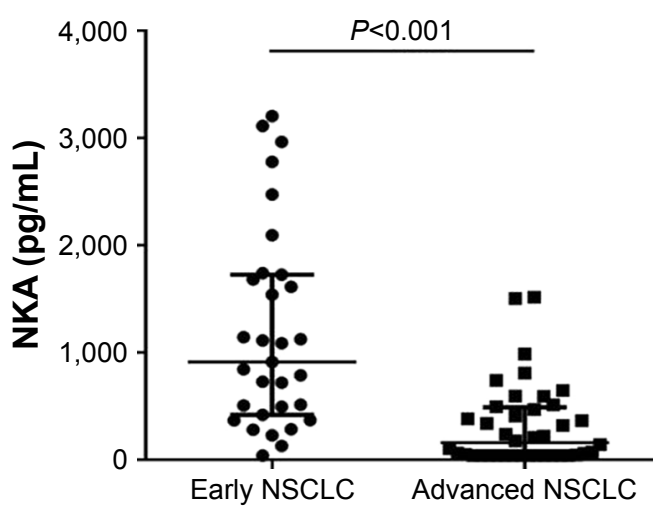

Figure 2 Comparison of NKA according to the stage of NSCLC.

Notes: NKA in stage III or IV was significantly lower than that in stage I or II (all $P<0.05)$. There was no significant difference between stage I and II or stage III and IV (A). NKA was significantly lower in those with advanced (stage III and IV) NSCLC than in those with early-stage (stage I and II) NSCLC (P<0.00I) (B).

Abbreviations: NKA, natural killer cell activity; NSCLC, non-small cell lung cancer. 
Table 2 Association between clinical parameters and NKA

\begin{tabular}{l|l|l|l|l|l|l}
\hline & \multicolumn{2}{l|}{} & \multicolumn{3}{l}{ Multivariate analysis } \\
\cline { 2 - 7 } & $\boldsymbol{\beta}^{\mathbf{a}}$ & $\mathbf{9 5 \%} \mathbf{C l}$ & $\boldsymbol{P}$-value & $\boldsymbol{\beta}^{\text {a }}$ & $\mathbf{9 5 \%} \mathbf{C l}$ & $\boldsymbol{P}$-value \\
\hline Male (vs female) & -270.0 & -593.0 to 52.9 & 0.101 & -322.5 & -713.2 to 62.4 & 0.107 \\
Age, years $\geq 65$ (vs $<65$ ) & -252.7 & -565.3 to 59.5 & 0.118 & -80.9 & -383.5 to 221.7 & 0.596 \\
Ever smoking (vs never smoking) & -280.7 & -691.6 to 129.8 & 0.176 & 23.3 & -432.1 to -478.7 & 0.919 \\
Benign lung disease (vs without & -46.7 & -442.1 to 348.7 & 0.215 & -39.4 & -456.4 to 377.6 & 0.852 \\
benign lung disease) & & & & & & \\
NSCLC (vs without NSCLC) & -826.7 & $-1,176.3$ to -447.1 & $<0.001$ & -833.4 & $-1,212.3$ to -455.6 & $<0.00$ I \\
\hline
\end{tabular}

Note: ${ }^{a} \beta$, beta coefficient.

Abbreviations: NKA, natural killer cell activity; NSCLC, non-small cell lung cancer.

useful method because it can estimate cytotoxicity and cytokine production activity of NK cells. The method used in the current study is a novel assay to evaluate NKA in a simple and standardized manner. Following in vitro stimulation using a patented recombinant protein, it measures concentration of IFN- $\gamma$ as a surrogate marker for NKA. Although NK cells are known to be the only cells that can produce this cytokine in whole blood upon stimulation, possible contribution of other immune cells during this assay should be taken into account. To eliminate this concern, a recent study has analyzed the distribution of IFN- $\gamma$-expressing lymphocyte subsets and verified that NK cells are predominant source of IFN- $\gamma$ in this assay suggesting the result per se reflects NKA. ${ }^{21}$ Our present study clearly showed decreased NKA measured by this novel assay in NSCLC patients, consistent with previous studies that measured NKA using traditional methods. ${ }^{18,22}$ Taken together, this assay can be a simple and feasible method to evaluate NKA in daily clinical practice. Although large-scaled studies are required to validate our results, the present data suggest potential usefulness of this assay to support lung cancer diagnosis.
We observed profound decease in NKA in patients with advanced NSCLC compared with that in those with earlystage disease, consistent with previous reports on other solid tumors. ${ }^{13-15}$ Compromised NKA in advanced diseases shown in previous data and our results reflects NK cell dysfunction and impairment of host's immune surveillance, where cancer cells readily metastasize to lymph nodes or other organs. Mechanism of decreased NKA in malignancy is not fully understood yet. However, previous studies have suggested that it is not due to decreased number of NK cells, but attributable to impaired immune regulatory system in cancer patients. ${ }^{22,23}$ In addition, humoral factors, such as tumor growth factor-beta1 released from tumor-associated monocyte/macrophage have been suggested to be associated with NK cell dysfunction. ${ }^{23}$ Moreover, reduced CD16 ${ }^{-}$ CD56 $6^{\text {bright }} \mathrm{NK}$ cell subset as major source of IFN- $\gamma$ has been suggested as another mechanism. ${ }^{19}$ In the present study, we further assessed the ratio of $\mathrm{CD} 16^{+} \mathrm{CD} 56^{\mathrm{dim}} / \mathrm{CD} 16^{-} \mathrm{CD} 56^{\text {bright }}$ NK cells to investigate whether this ratio was different among study groups and whether NKA was affected by this ratio. Our data showed that the ratio was not different among
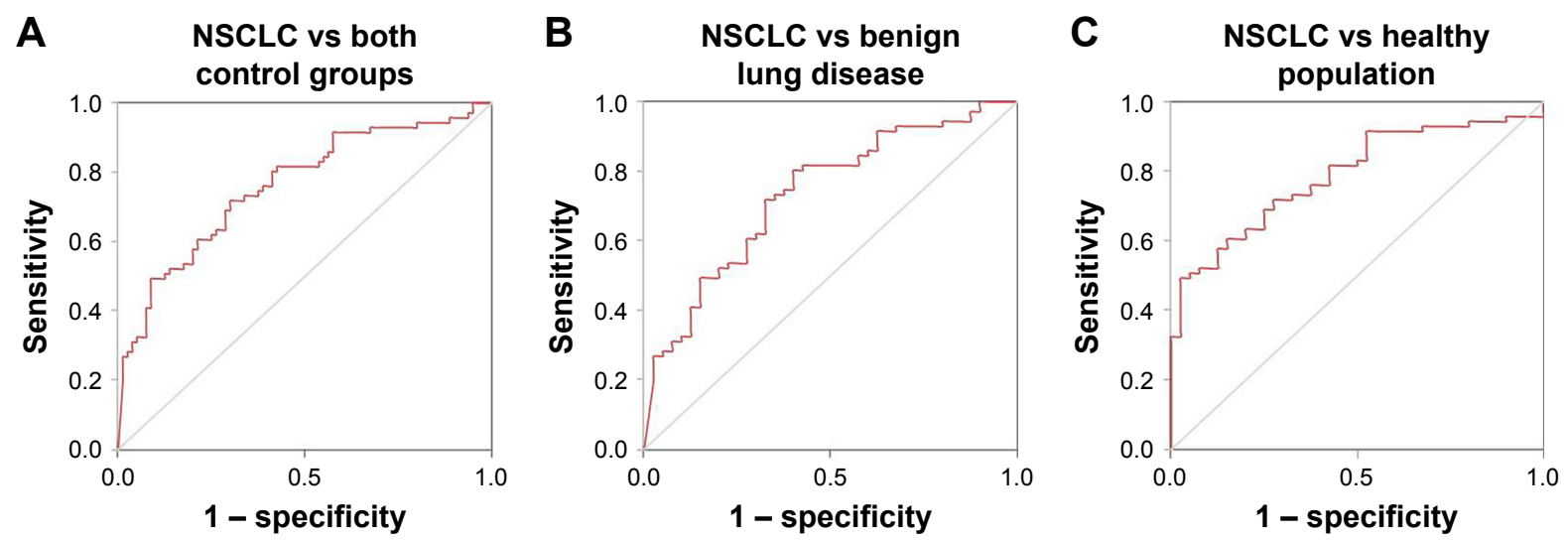

Figure 3 Receiver operating characteristic curve analysis of NKA for the diagnosis of NSCLC being control groups (A), benign lung disease (B) and healthy population (C) as references. The AUCs ranged from 0.735 to 0.789 with considerable sensitivity and specificity (all $P<0.00 \mathrm{I}$ ). Based on AUC value, the best diagnostic performance of NKA was noted when the reference was the healthy population.

Abbreviations: AUC, area under the curve; NKA, natural killer cell activity; NSCLC, non-small cell lung cancer. 
groups and it was not correlated with NKA. These findings suggest that the difference in NKA among our study groups was not due to change of NK cells subset distributions but rather reflects cytokine-producing capacity of both subsets of NK cells. Furthermore, our data suggest that measurement of NKA rather than NK cells subset ratio can be a feasible method that can support lung cancer diagnosis.

Our data showed that NKA varied with stage of NSCLC. Although there was no significant difference in NKA between two groups, a decreasing tendency was shown in early-stage NSCLC patients compared with control groups (912.7 vs $1,372.3 \mathrm{pg} / \mathrm{mL}, P=0.08)$. Subsequent studies need to assess possible clinical implication of NKA in early-stage NSCLC. At this point, NKA alone seems to be unsuitable for screening or detecting early lung cancer. However, NKA could be used as a surveillance method after systemic treatment in advanced diseases because compromised NKA implies evasion of antitumor immune response and progression of tumors. Future studies should focus on serial changes of NKA during the clinical course and the possible implication of NKA-based monitoring during lung cancer treatment. Despite the weak relevance of NKA in early-stage NSCLC, clinical utility of NKA measurement in high-risk smokers who undergo lung cancer screening and the discriminative power of NKA for differentiating malignant nodules from benign ones would be feasible topics for future investigations. Although there are scarce data on the implication of NKA in patients with precancerous or early-stage malignant lesions, our hypotheses are supported by a recent study demonstrating significantly compromised NKA in subjects with colorectal adenomas compared with those without such lesions in colonoscopy. ${ }^{24}$

In the present study, diagnosis of NSCLC was the only clinical parameter associated with NKA. Although there are some conflicting data, human NKA does not seem to be affected by age or gender. ${ }^{25}$ In terms of smoking, a few in vitro or in vivo studies have suggested that cigarette smoking may be associated with low NKA. ${ }^{26,27}$ A small human study on the effect of life style on individual NKA has reported similar results. ${ }^{25}$ However, there was no report on the association between cigarette smoking and NKA in diseased population so far. In the present study, we could not find any association between smoking status and NKA either in each study group or in whole study population analyzed by multivariable analysis. Although sample size is small, we can cautiously postulate that adverse effect of smoking on NKA can vary according to individuals' health status and NKA may not be affected by smoking status at least in patients with lung cancer. The association between smoking and NKA in the population with medical illness, and the possible difference of smoking effect on NKA between patients with benign and malignant disease should be a topic of future studies.

This study has several limitations. First, this was a small explorative study performed in a single institution without matching clinical factors, such as age and sex among groups. To make up for the small sample size, we enrolled subjects prospectively and included patients with benign lung disease as a control along with healthy population. Second, we did not simultaneously evaluate or compare diagnostic performance of other biomarkers for lung cancer. Comparison of diagnostic value and evaluation of combined effect with established biomarkers, including carcinoembryonic antigen and cytokeratin 19 would provide more informative data. Finally, we did not evaluate the prognostic value of NKA in NSCLC patients. To that end, we are currently performing prospective studies to evaluate the impact of NKA on tumor recurrence or survival in early-stage lung cancer patients who received surgical resection and its impact on clinical outcome in advanced NSCLC patients treated with various systemic treatments.

\section{Conclusion}

In summary, we verified that NKA was reduced in patients with NSCLC compared with that in cancer-free population. NKA was compromised more in those with advanced NSCLC than in those with early-stage disease, reflecting more profound immune escape of lung cancer cells in the former. Our data suggest the clinical implication of NKA measurement using a simple blood test and the usefulness of NKA as a potential supportive method for lung cancer diagnosis. Further validation of our results in large population and identification of prognostic value of NKA are clearly required. Our pilot results provide a basis for future investigation on NKA in various clinical settings for lung cancer management, including complementary lung cancer screening method among high-risk smokers and discrimination of individuals with high recurrence risk after surgery, which might facilitate the clinical use of this novel assay.

\section{Acknowledgment}

This work was supported by a grant (NRF-2017R1C1B 5016828) from the National Research Foundation (NRF) funded by the Ministry of Science and ICT, South Korea.

\section{Disclosure}

The authors report no conflicts of interest in this work. 


\section{References}

1. Vivier E, Raulet DH, Moretta A, et al. Innate or adaptive immunity? The example of natural killer cells. Science. 2011;331(6013):44-49.

2. De Maria A, Bozzano F, Cantoni C, Moretta L. Revisiting human natural killer cell subset function revealed cytolytic CD56(dim)CD16+ NK cells as rapid producers of abundant IFN-gamma on activation. Proc Natl Acad Sci U S A. 2011;108(2):728-732.

3. Bauernhofer T, Kuss I, Henderson B, Baum AS, Whiteside TL. Preferential apoptosis of CD56dim natural killer cell subset in patients with cancer. Eur J Immunol. 2003;33(1):119-124.

4. Takeuchi H, Maehara Y, Tokunaga E, Koga T, Kakeji Y, Sugimachi K. Prognostic significance of natural killer cell activity in patients with gastric carcinoma: a multivariate analysis. Am J Gastroenterol. 2001;96(2): 574-578.

5. Kim S, Iizuka K, Aguila HL, Weissman IL, Yokoyama WM. In vivo natural killer cell activities revealed by natural killer cell-deficient mice. Proc Natl Acad Sci U S A. 2000;97(6):2731-2736.

6. Konjević G, Spuzić I. Stage dependence of NK cell activity and its modulation by interleukin 2 in patients with breast cancer. Neoplasma. 1993;40(2):81-85.

7. Tarle M, Kraljić I, Kastelan M. Comparison between NK cell activity and prostate cancer stage and grade in untreated patients: correlation with tumor markers and hormonal serotest data. Urol Res. 1993;21(1): 17-21.

8. Hanna N, Burton RC. Definitive evidence that natural killer (NK) cells inhibit experimental tumor metastases in vivo. J Immunol. 1981;127(5): 1754-1758.

9. Steinhauer EH, Doyle AT, Reed J, Kadish AS. Defective natural cytotoxicity in patients with cancer: normal number of effector cells but decreased recycling capacity in patients with advanced disease. J Immunol. 1982;129(5):2255-2259.

10. Imai K, Matsuyama S, Miyake S, Suga K, Nakachi K. Natural cytotoxic activity of peripheral-blood lymphocytes and cancer incidence: an 11-year follow-up study of a general population. Lancet. 2000; 356(9244):1795-1799.

11. Valiathan R, Lewis JE, Melillo AB, Leonard S, Ali KH, Asthana D. Evaluation of a flow cytometry-based assay for natural killer cell activity in clinical settings. Scand J Immunol. 2012;75(4):455-462.

12. Lee SB, Cha J, Kim IK, et al. A high-throughput assay of NK cell activity in whole blood and its clinical application. Biochem Biophys Res Commun. 2014;445(3):584-590.

13. Koo KC, Shim DH, Yang CM, et al. Reduction of the CD16(-) CD56bright NK cell subset precedes NK cell dysfunction in prostate cancer. PLoS One. 2013;8(11):e78049.
14. Barkin J, Rodriguez-Suarez R, Betito K. Association between natural killer cell activity and prostate cancer: a pilot study. Can J Urol. 2017; 24(2):8708-8713.

15. Lee J, Park KH, Ryu JH, et al. Natural killer cell activity for IFNgamma production as a supportive diagnostic marker for gastric cancer. Oncotarget. 2017;8(41):70431-70440.

16. Jobin G, Rodriguez-Suarez R, Betito K. Association between natural killer cell activity and colorectal cancer in high-risk subjects undergoing colonoscopy. Gastroenterology. 2017;153(4):980-987.

17. Villegas FR, Coca S, Villarrubia VG, et al. Prognostic significance of tumor infiltrating natural killer cells subset CD57 in patients with squamous cell lung cancer. Lung Cancer. 2002;35(1):23-28.

18. Al Omar SY, Marshall E, Middleton D, Christmas SE. Increased killer immunoglobulin-like receptor expression and functional defects in natural killer cells in lung cancer. Immunology. 2011;133(1):94-104.

19. Poli A, Michel T, Thérésine M, Andrès E, Hentges F, Zimmer J. CD56bright natural killer (NK) cells: an important NK cell subset. Immunology. 2009;126(4):458-465.

20. Phan MT, Chun S, Kim SH, et al. Natural killer cell subsets and receptor expression in peripheral blood mononuclear cells of a healthy Korean population: reference range, influence of age and sex, and correlation between NK cell receptors and cytotoxicity. Hum Immunol. 2017;78(2):103-112.

21. Nederby L, Jakobsen A, Hokland M, Hansen TF. Quantification of NK cell activity using whole blood: methodological aspects of a new test. J Immunol Methods. 2018;458:21-25.

22. Ciszak L, Kosmaczewska A, Werynska B, Szteblich A, Jankowska R, Frydecka I. Impaired zeta chain expression and IFN-gamma production in peripheral blood T and NK cells of patients with advanced lung cancer. Oncol Rep. 2009;21(1):173-184.

23. Peng LS, Zhang JY, Teng YS, et al. Tumor-associated monocytes/ macrophages impair NK-cell function via TGF $\beta 1$ in human gastric cancer. Cancer Immunol Res. 2017;5(3):248-256.

24. Jung YS, Kwon MJ, Park DI, Sohn CI, Park JH. Association between natural killer cell activity and the risk of colorectal neoplasia. J Gastroenterol Hepatol. 2018;33(4):831-836.

25. Morimoto K, Takeshita T, Inoue-Sakurai C, Maruyama S. Lifestyles and mental health status are associated with natural killer cell and lymphokine-activated killer cell activities. Sci Total Environ. 2001; 270(1-3):3-11.

26. Lu LM, Zavitz CC, Chen B, Kianpour S, Wan Y, Stämpfli MR. Cigarette smoke impairs NK cell-dependent tumor immune surveillance. J Immunol. 2007;178(2):936-943.

27. Mian MF, Lauzon NM, Stämpfli MR, Mossman KL, Ashkar AA. Impairment of human NK cell cytotoxic activity and cytokine release by cigarette smoke. J Leukoc Biol. 2008;83(3):774-784. 


\section{Supplementary materials}
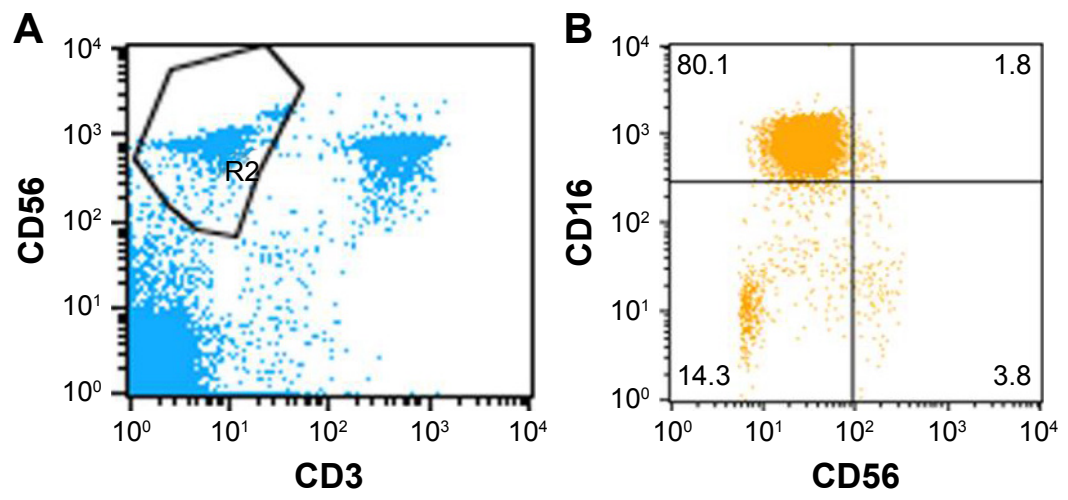

Figure SI Representative flow cytometric data showing the distribution of NK cell subsets.

Notes: Total NK cell population represented as CD3-CD56 cells NK cells (A) are gated into two subsets, CDI6 $6^{+} \mathrm{CD} 56^{\text {dim }}$ and $C D 16^{-} \mathrm{CD} 56^{\text {bright }}$, according to intensity of CDI6 and CD56 expression (B). CD I6 ${ }^{+}$CD $56^{\text {dim }}$ NK cell subset comprise major subset (left upper box) while CDI6-CD56 bright (right lower box) comprise minor subset.

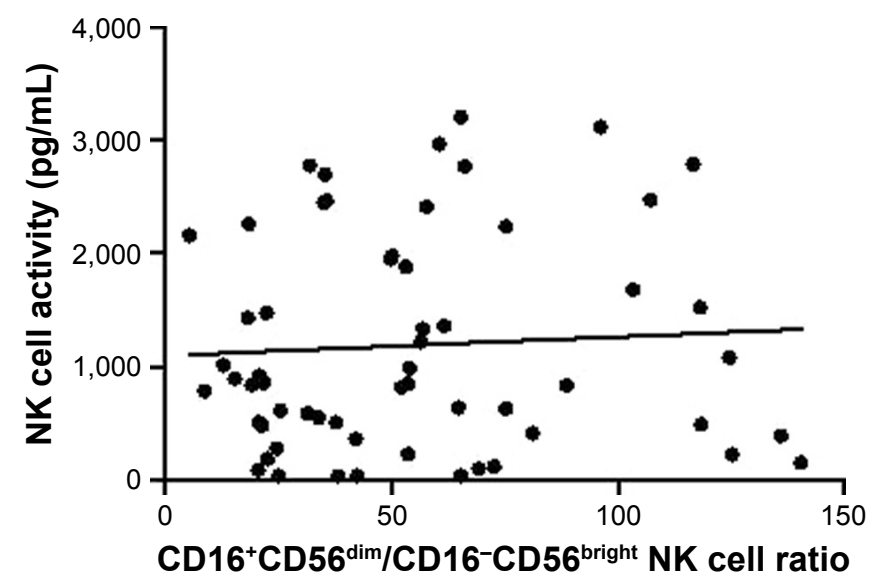

Figure S2 Overall relationship between the NK cell activity and the CDI6+CD56 $6^{\mathrm{dim}} / \mathrm{CD} 16^{-} \mathrm{CD} 56^{\text {bright }} \mathrm{NK}$ cell ratio.

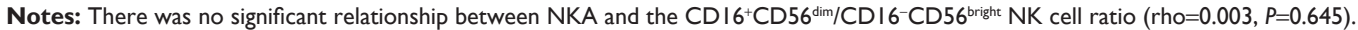

Abbreviation: NK, natural killer.

\section{Publish your work in this journal}

OncoTargets and Therapy is an international, peer-reviewed, open access journal focusing on the pathological basis of all cancers, potential targets for therapy and treatment protocols employed to improve the management of cancer patients. The journal also focuses on the impact of management programs and new therapeutic agents and protocols on patient perspectives such as quality of life, adherence and satisfaction. The manuscript management system is completely online and includes a very quick and fair peer-review system, which is all easy to use. Visit http://www.dovepress.com/testimonials.php to read real quotes from published authors. 